\title{
First step toward uncovering perioperative congestive encephalopathy
}

William Beaubien-Souligny, MD, ,

Yiorgos Alexandros Cavayas, MD, MSc, ${ }^{\mathrm{c}, \mathrm{d}}$

André Denault, $\mathrm{MD}, \mathrm{PhD},{ }^{\mathrm{a}, \mathrm{e}}$ and

Yoan Lamarche, MD, MSc ${ }^{\mathrm{c}, \mathrm{f}}$

Supplemental material is available online.

Continuous advances in perioperative care during past decades resulted in a substantial decline in mortality rate after cardiac surgery. However, acute complications such as postoperative delirium (POD) still occur and do so with a high frequency in elderly patients undergoing cardiac surgery. ${ }^{1}$ The burden of POD is both important from the perspective of the patient and of the healthcare system. This complication is associated with adverse outcomes, including patientcentered outcomes such as discharge to a nonhome health care facility, a decline in functional status, and increased risk of subsequent postoperative neurocognitive disorder. ${ }^{2,3}$ Clinicians often feel powerless when confronted with persistent postoperative delirium. The precise mechanism that leads to this state is often not readily apparent. Although there are considerable data suggesting that the systemic inflammatory response triggered by cardiopulmonary bypass may directly induce cognitive dysfunction, ${ }^{4}$ a myriad of intraoperative and post-operative factors may be contributors to variable degrees. However, it might be challenging to detect them and determine which are clinically significant enough to merit intervention. When the arterial blood pressure targets are met, blood gases are satisfactory, and the cardiac output appears adequate, what can be done to improve patients who remain confused? We have yet to find an intervention to reliably prevent or treat

\footnotetext{
From the Departments of ${ }^{\mathrm{a}}$ Anesthesiology and ${ }^{\mathrm{c}}$ Cardiac Surgery, Intensive Care Unit, Montreal Heart Institute, Université de Montréal, Montreal, Québec, Canada; Divisions of ${ }^{\mathrm{b}} \mathrm{Nephrology}$ and ${ }^{\mathrm{e}}$ Intensive Care, Centre Hospitalier de l'Université de Montréal, Montreal, Québec, Canada; and Departments of ${ }^{\mathrm{d}}$ Medicine and ${ }^{\mathrm{f}}$ Cardiac Surgery, Hôpital Sacré-Coeur de Montréal, Montreal, Québec, Canada.

Received for publication Nov 12, 2019; revisions received Feb 5, 2020; accepted for publication Feb 5, 2020; available ahead of print July 2, 2020.

Address for reprints: Yoan Lamarche, MD, MSc, Montreal Heart Institute, 5000 rue Belanger, Montreal, Québec H1T 1C8, Canada (E-mail: yoanlamarche@gmail. com).

J Thorac Cardiovasc Surg 2021;161:149-53 0022-5223/\$36.00

Copyright (c) 2020 Published by Elsevier Inc. on behalf of The American Association for Thoracic Surgery

https://doi.org/10.1016/j.jtcvs.2020.02.146
}

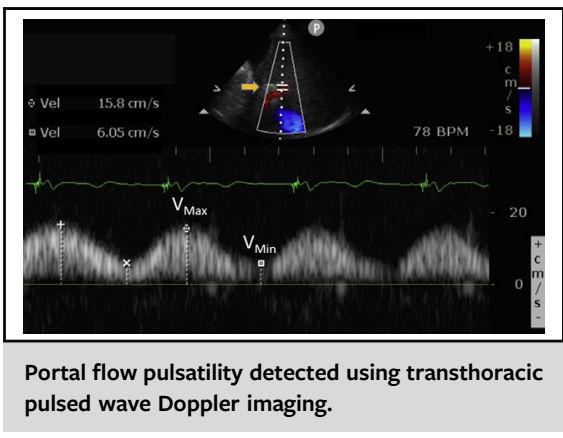

CENTRAL MESSAGE

Cerebral venous congestion could be a mechanism leading to delirium in some patients after cardiac surgery. Portal flow assessment is a promising tool to identify significant congestion.

This Invited Expert Opinion provides a perspective on the following paper: Can J Cardiol. 2019;35(9):1134-1141 2019.04.006

See Commentary on page 154.

POD in all cardiac surgery patients. ${ }^{5}$ This may result from our inability to reliably identify major contributors to cognitive dysfunction that may vary greatly between individuals. Precision medicine precepts holds that identifying subphenotypes with common biologic contributors could allow us to study and apply more specific management strategies. ${ }^{6}$

\section{AN OVERLOOKED THREAT}

The concept of detrimental venous congestion is not new. Animal experiments dating back to 1913 demonstrated the progressive impairment of mammalian kidney function upon a rise in the pressure in the renal vein in a controlled setting. ${ }^{7}$ More recent evidence provides compelling arguments regarding similar adverse effects on the liver, ${ }^{8}$ the gastrointestinal tract, ${ }^{9,10}$ soft tissues, ${ }^{11,12}$ and the myocardium. ${ }^{13,14}$ Data on how venous congestion might affect the brain are scarcer. However, it is unlikely that an organ contained in a nonexpandable cavity (eg, the cranium) could be the sole left unaffected by interstitial edema, 
particularly in the setting of prolonged recumbent position necessary in the perioperative period. Subclinical cerebral edema has been shown to occur in a proportion of patients undergoing cardiac surgery. ${ }^{15}$ Additionally, the release of blood-brain biomarkers suggesting subclinical cerebral injury has been described in patients with disturbance of venous outflow resulting in high jugular venous bulb pressure $\left(>12 \mathrm{~mm} \mathrm{Hg}\right.$ ) during surgery. ${ }^{16}$ The degree of cerebral venous pressure elevation is correlated with intraabdominal pressure and central venous pressure (CVP) ${ }^{17}$ Data from an animal model of intra-abdominal hypertension suggests that the increase in jugular venous pressure in this setting may induce histologic changes suggestive of cerebral ischemia. ${ }^{18}$ Furthermore, the influence of cerebral congestion has been noted in other settings. Neurocognitive outcomes appear to be worse in critically ill patients with evidence of venous congestion after cardiac arrest ${ }^{19}$ or with a significant positive fluid balance in the setting of shock. ${ }^{20}$ Despite these preliminary findings, much is left to learn because this mechanism of brain injury has largely been overlooked so far.

Undetected venous congestion may contribute significantly to cognitive dysfunction in a subgroup of patients undergoing cardiac surgery. Although higher CVPs have been associated with an increased risk of complications in multiple acute care settings, including cardiac surgery, ${ }^{21}$ the CVP threshold above which harm occurs remains uncertain. This might be in part due to imprecisions in CVP monitoring, particularly in critical care settings. Accurate CVP measurements rely on adequate transducer placement and minor variations can result in important discrepancies, even in experienced operators. ${ }^{22}$ Furthermore, invasive monitoring is discontinued early during the postoperative course and may be unavailable when POD becomes apparent, usually from 24 to 72 hours after cardiac surgery.

Apart from the potential direct effect of venous congestion on the brain through the development of interstitial edema and increased intracranial pressure, venous congestion of other organs might contribute to cognitive dysfunction (Figure 1,A). The development of elevated intra-abdominal pressure is a well-known consequence of congestive heart failure, as well as fluid overload in critically ill patients, and may contribute to elevated intracranial pressure as previously presented. ${ }^{23}$ Furthermore, intestinal edema may be an important contributor to the general inflammatory reaction seen in cardiac surgery patients through the dysfunction of the intestinal barrier function leading to endotoxin translocation. This phenomenon, colloquially known as cardiointestinal syndrome, (Table E1) has been described in congestive heart failure patients but may also occur in acute settings. Whereas the link between unabated inflammation process and cognitive dysfunction after cardiac surgery is well documented, the contribution of endogenous endotoxemia is not documented. Finally, in extreme cases, congestive liver and kidney function impairment may contribute through hepatic and uremic encephalopathy. Portal vein flow pulsatility may be a practical marker of relative cardiogenic portal hypertension able to identify patients at risk for developing these phenomena. ${ }^{24}$

\section{A NEW MODALITY}

Assessing the influence of venous congestion directly in end organs by a noninvasive method might be an important adjunct to correctly identify a congestive phenotype. Distension modifies the properties of the systemic venous system, which changes from a highly compliant structure to a more rigid network. Pressure variations in the right atrium are usually poorly transmitted distally due to high damping. However, they are readily transmitted in a distended, stiff system. Point-of-care ultrasonography enables clinicians to assess venous blood velocity directly into end organs using Doppler imaging. ${ }^{25}$ Using this modality, it is possible to detect characteristic distal pulsatile venous waveforms suggesting decreased systemic venous compliance. Portal vein flow is perhaps the most reliable window to assess the compliance of the venous system. Portal flow pulsatility is defined as a difference greater than $30 \%$ to $50 \%$ between the maximum and minimum Doppler flow velocities measured in the main portal vein during the cardiac cycle (Figure $1, B) .{ }^{26}$ Originally described in congestive heart failure patients with right heart failure and/or tricuspid regurgitation, it has been shown to be the best sonographic predictor of a cholestatic liver profile in this population. ${ }^{27}$ Notably, it was superior to other complex echocardiography measurements of right ventricular function and pressure. ${ }^{27}$ This suggest that, in addition to being less technically complex, assessing the hemodynamic influence on portal blood flow might be a better congestion marker than traditional echocardiographic assessment of right ventricular function. More recently, we have shown that portal pulsatility is detected in an important proportion ( $\approx 20 \%-25 \%$ ) of the cardiac surgery population, including during surgery ${ }^{28}$ and in the days following cardiac surgery, ${ }^{29}$ with a peak on postoperative day 2 after surgery. The magnitude of pulsatility is correlated with right ventricular diastolic pressure during surgery ${ }^{28}$ and natriuretic peptide after surgery ${ }^{29}$ strengthening its potential role as a congestion biomarker in cardiac surgery populations. In a first prospective cohort study $(\mathrm{n}=115)$, the detection of portal flow pulsatility by transesophageal echocardiography at the end of surgery was independently associated with major complications (odds ratio [OR], 5.13; 95\% confidence interval $[\mathrm{CI}], 1.58-16.67 ; P=.007)$. In a second prospective cohort study $(\mathrm{n}=145)$, portal flow pulsatility detected during the first 3 days after cardiac surgery was independently associated with acute kidney injury and renal venous flow alterations. The success rate of portal flow assessment 

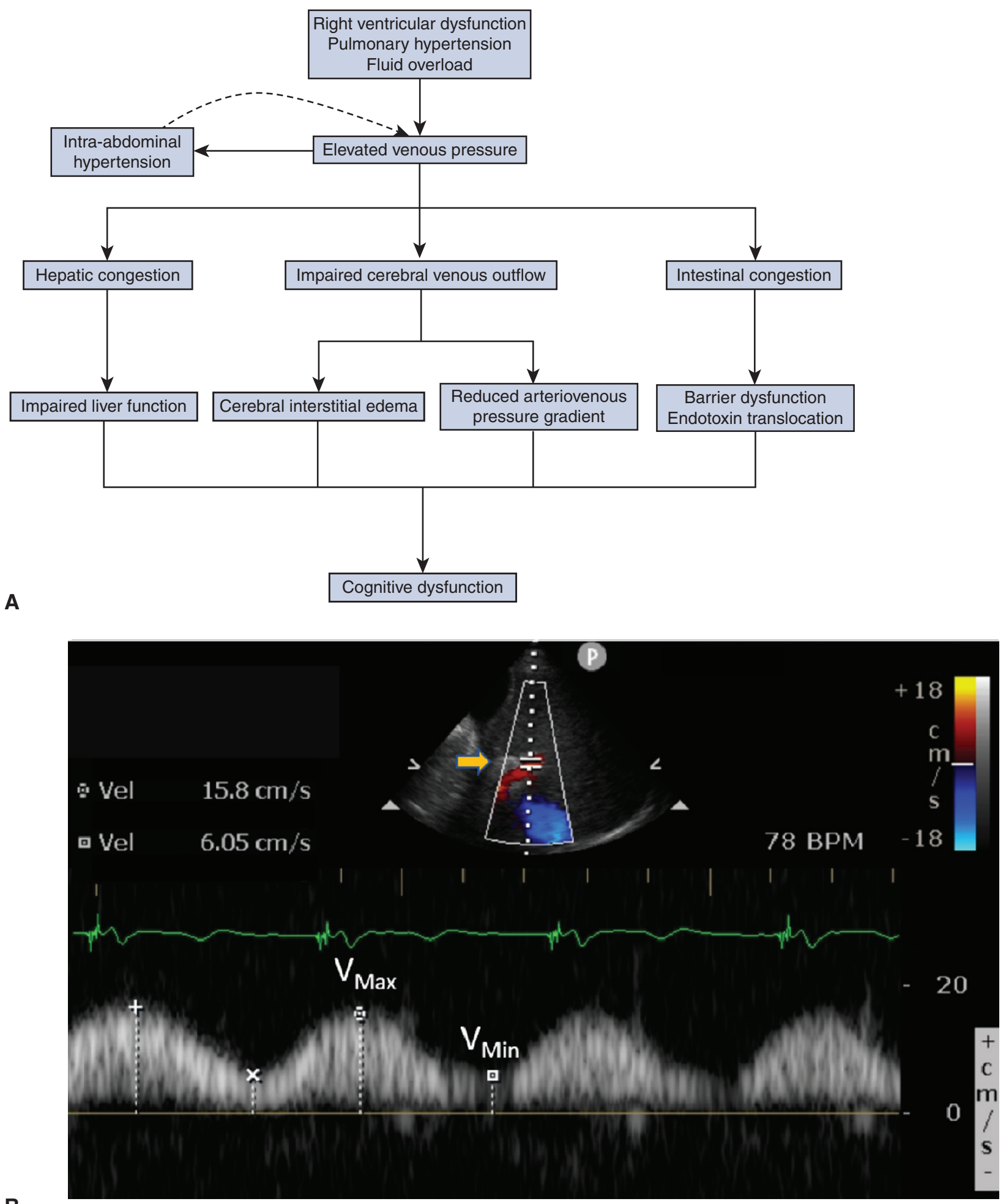

\section{B}

FIGURE 1. A, Potential mechanisms linking systemic venous congestion to postoperative delirium. B, Portal flow pulsatility in a patient aged 79 years detected 3 days after mitral valve replacement. Variations in blood velocity within the portal vein are optimally assessed at end expiration at the hepatic ilium where the vessel enters the liver (orange arrow) using a cardiac probe (phased-array transducer) or an abdominal probe (curved array transducer) using pulsed wave Doppler. The concurrent echocardiogram tracing enables the identification of the minimal $\left(V_{\text {Min }}\right)$ and maximal $\left(V_{\text {Max }}\right)$ velocity during the cardiac cycle. Portal pulsatility fraction (PF) can be quantified as following: $\left[\mathrm{V}_{\mathrm{Max}}-\mathrm{V}_{\mathrm{Min}}\right] / \mathrm{V}_{\mathrm{Max}}(\mathrm{PF}, 62 \%$ is shown). $\mathrm{PF}$ of $30 \%$ up to $50 \%$ is considered mild pulsatility, whereas $\mathrm{PF}>50 \%$ is considered severe pulsatility. At the time of assessment, the patient was disoriented and presented asterixis. He had a weight gain of $2.6 \mathrm{~kg}$ compared with preoperative baseline, a N-terminal-pro beta-natriuretic peptide measurement of $3474 \mathrm{pg} / \mathrm{mL}$ (preoperative level, $1060 \mathrm{pg} / \mathrm{mL}$ ) and a brain oxygen saturation of $52 \%$ representing a $21 \%$ relative decrease from preoperative baseline. Vel, Velocity. 
was $94 \%$ to $95 \%$ by transesophageal echocardiography ${ }^{28}$ and $98 \%$ to $99 \%$ by transthoracic echocardiography ${ }^{28,29}$ with a good interobserver agreement (mean absolute percentage difference, $6.1 \% \pm 6.2 \%$ ) and a very good intraclass correlation coefficient $(0.95 ; P<.001){ }^{29}$

In a prespecified substudy, the detection of portal flow pulsatility was associated with cognitive dysfunction (OR, $2.10 ; 95 \% \mathrm{CI}, 1.25-3.53 ; P=.005)$ and asterixis (OR, 2.23 ; 95\% CI, 1.13-4.41; $P=.02)$ assessed by the investigators as well as POD detected by the nursing staff during regular screening (hazard ratio, 2.63; 95\% CI, 1.13-6.11; $P=.025) .{ }^{30}$ Of note, patients with portal flow pulsatility were more likely to present significantly lower cerebral tissue oximetry at the time of assessment compared with preoperative baseline (OR, 2.23; 95\% CI, 1.12-4.71; $P=.02$ ). Whether this finding was related to the greater proportion of venous blood in the cerebral circulation or to a reduction of cerebral perfusion due to elevated intracranial pressure is not known. These observations are the first to report the association between an echocardiography marker of congestion and cognitive dysfunction in a cohort of patients undergoing cardiac surgery.

\section{A WAY FORWARD}

These novel findings should be interpreted with caution. A multicenter cohort study is currently ongoing to confirm the association found between portal flow pulsatility and postoperative complications, including delirium (ClinicalTrials.gov identifier: NCT03656263). Nevertheless, validating this finding is only the first step in determining whether interventions targeted at restoring normal venous compliance in response to the detection of portal flow pulsatility may contribute to prevent POD or treat a subgroup of patients with congestive POD. An overview of the critical time points where portal flow assessment may be warranted is presented in Figure 2. Preventive strategies to avoid congestive POD could be first related to achieving an optimal fluid balance status before surgery in patients with pre-existing congestive heart failure who present with a pulsatile portal vein flow during the preoperative consultation. Secondly, detecting a pulsatile portal flow by transesophageal echocardiography at the end of surgery or at intensive care unit admission might represent a second opportunity to prevent congestive organ injury. Interventions that may accomplish that goal may include the induction of a negative fluid balance, the pre-emptive use of renal replacement therapy when significant renal dysfunction is present, or the use of inhaled or intravenous inotropes to improve heart function and right ventricular afterload. The best strategy may vary according to patient status at the time of assessment. Finally, portal vein flow assessment may be performed in patients presenting POD to determine if venous congestion might contribute to cognitive dysfunction, which may respond to the aforementioned decongestion strategies.

Each of these clinical questions represents an indication to perform a different clinical trial to determine whether an ultrasound-based approach integrating portal vein Doppler would contribute to improve the care of patients undergoing cardiac surgery. Numerous challenges are foreseen when considering such types of interventional studies. These include defining the nature of the proposed intervention and whether it will be appropriate in most patients given the highly variable and rapidly evolving nature of postcardiac surgery care. Notably, it will be important to determine the potential situations in which portal vein Doppler may be misleading; to validate the optimal cutoff suggesting a pathologic process; and to determine how
Pre-operative detection: (Adding congestion evaluation to pre-operative risk assessment)
Early detection: (Identifying a risk of congestive complications during or shortly after surgery)
Late detection: (Identifying congestion as a potential

contributor to ongoing organ injury)
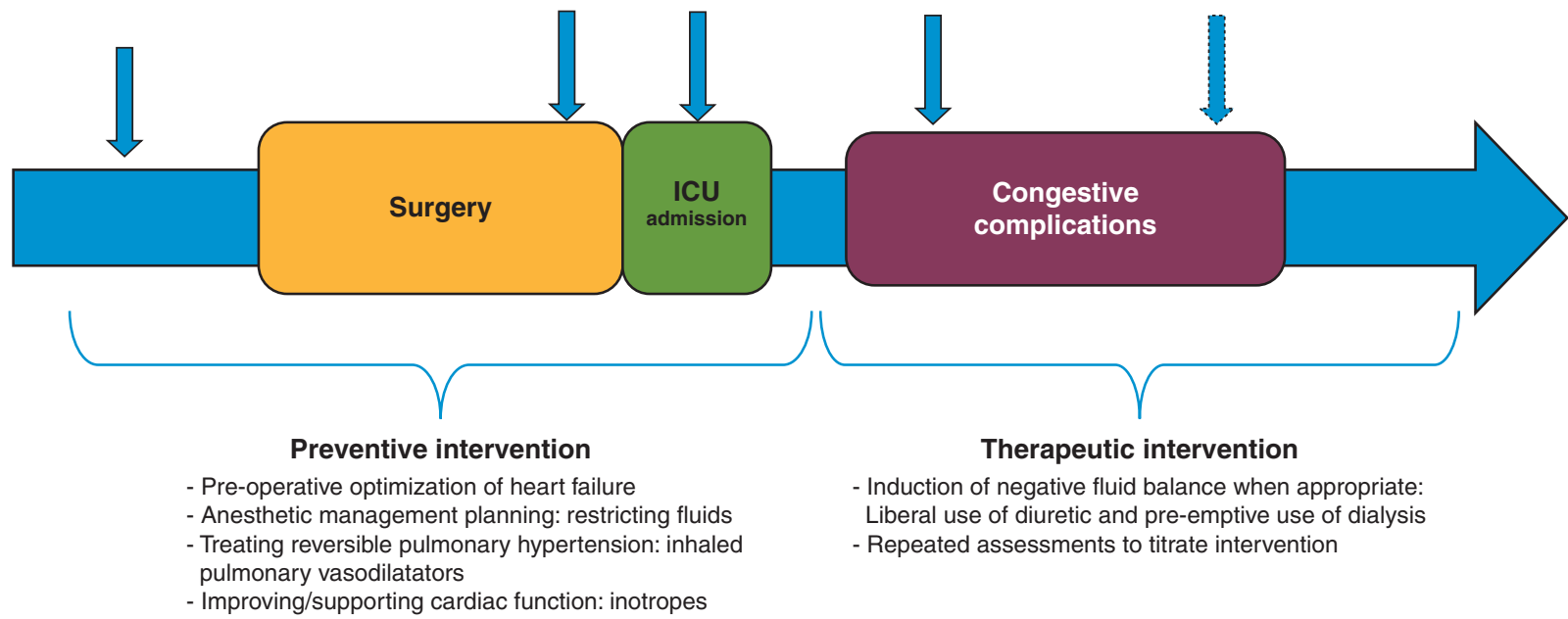

Therapeutic intervention

- Induction of negative fluid balance when appropriate: Liberal use of diuretic and pre-emptive use of dialysis

- Repeated assessments to titrate intervention pulmonary vasodilatators

- Improving/supporting cardiac function: inotropes

FIGURE 2. Potential preventive and therapeutic applications of assessing venous congestion in the perioperative period. ICU, Intensive care unit. 
combining it to other echocardiography, clinical, and laboratory variables might improve interpretation. Ultrasoundguided interventions also necessitate a critical mass of trained ultrasound operators to perform the assessments. Fortunately, portal vein Doppler is a reliable technique that may be rapidly learned by operators with intermediate ultrasound skills. It is likely that this level of competency will be considered standard in the future given the current growth of enthusiasm for point-of-care ultrasound within the acute cardiac care community, opening opportunities to study the influence of ultrasound-guided strategies.

Preliminary data suggest that portal flow Doppler could be a simple noninvasive tool to detect clinically significant venous congestion at the bedside of cardiac surgery patients. Whether or not portal flow Doppler is confirmed to be the optimal clinical tool, clinicians should be aware that venous congestion could be a major contributor to the high incidence of POD after cardiac surgery. Unraveling congestive brain injury may enable clinicians to treat the cause of POD in selected patients rather than merely controlling the symptoms.

\section{Conflict of Interest Statement}

Dr Beaubien-Souligny has received salary support from Fonds de Recherche du Québec en Santé. Dr Denault is supported by the Montreal Heart Institute Foundation and the Richard Kaufman endowment fund in anesthesiology and critical care and is on the speakers bureau for CAE Healthcare and Masimo. All other authors reported no conflicts of interest.

The Journal policy requires editors and reviewers to disclose conflicts of interest and to decline handling or reviewing manuscripts for which they may have a conflict of interest. The editors and reviewers of this article have no conflicts of interest.

\section{References}

1. O'Brien H, Mohan H, Hare CO, Reynolds JV, Kenny RA. Mind over matter? The hidden epidemic of cognitive dysfunction in the older surgical patient. Ann Surg. 2017;265:677-91.

2. Gleason LJ, Schmitt EM, Kosar CM, Tabloski P, Saczynski JS, Robinson T, et al. Effect of delirium and other major complications on outcomes after elective surgery in older adults. JAMA Surg. 2015;150:1134-40.

3. Evered L, Silbert B, Knopman DS, Scott DA, DeKosky ST, Rasmussen LS, et al. Recommendations for the nomenclature of cognitive change associated with anaesthesia and surgery-2018. Can J Anaesth. 2018;65:1248-57.

4. Hogan AM, Shipolini A, Brown MM, Hurley R, Cormack F. Fixing hearts and protecting minds: a review of the multiple, interacting factors influencing cognitive function after coronary artery bypass graft surgery. Circulation. 2013;128: $162-71$.

5. Pieri M, De Simone A, Rose S, De Domenico T, Lembo R, Denaro G, et al. Trials focusing on prevention and treatment of delirium after cardiac surgery: a systematic review of randomized evidence. J Cardiothorac Vasc Anesth. 2020;34: 1641-54.

6. Vincent JL. The coming era of precision medicine for intensive care. Crit Care. 2017;21:314.

7. Rowntree LG, Fitz R, Geraghty JT. The effects of experimental chronic passive congestion on renal function. Arch Intern Med. 1913;XI:121-47.
8. Sundaram V, Fang JC. Gastrointestinal and liver issues in heart failure. Circulation. 2016;133:1696-703.

9. Niebauer J, Volk HD, Kemp M, Dominguez M, Schumann RR, Rauchlaus M, et al. Endotoxin and immune activation in chronic heart failure: a prospective cohort study. Lancet. 1999;353:1838-42.

10. Valentova M, von Haehling S, Bauditz J, Doehner W, Ebner N, Bekfani T, et al Intestinal congestion and right ventricular dysfunction: a link with appetite loss, inflammation, and cachexia in chronic heart failure. Eur Heart J. 2016;37: 1684-91.

11. Brandstrup B, Tonnesen H, Beier-Holgersen R, Hjortso E, Ording H, Lindorff-Larsen K, et al. Effects of intravenous fluid restriction on postoperative complications: comparison of two perioperative fluid regimens: a randomized assessor-blinded multicenter trial. Ann Surg. 2003;238:641-8.

12. Warrillow SJ, Weinberg L, Parker F, Calzavacca P, Licari E, Aly A, et al. Perioperative fluid prescription, complications and outcomes in major elective open gastrointestinal surgery. Anaesth Intensive Care. 2010;38:259-65.

13. Verbrugge FH, Bertrand PB, Willems E, Gielen E, Mullens W, Giri S, et al Global myocardial oedema in advanced decompensated heart failure. Eur Heart J Cardiovasc Imaging. 2017;18:787-94.

14. Madias JE. Apparent amelioration of bundle branch blocks and intraventricular conduction delays mediated by anasarca. J Electrocardiol. 2005;38:160-5.

15. Harris D, Bailey SM, Smith P, Taylor KM, Oatridge A, Bydder GM. Brain swelling in first hour after coronary artery bypass surgery. Lancet. 1993;342: 586-7.

16. Kotlinska-Hasiec E, Czajkowski M, Rzecki Z, Stadnik A, Olszewski K Rybojad B, et al. Disturbance in venous outflow from the cerebral circulation in tensifies the release of blood-brain barrier injury biomarkers in patients undergoing cardiac surgery. J Cardiothorac Vasc Anesth. 2014;28:328-35.

17. Dabrowski W. Changes in intra-abdominal pressure and central venous and brain venous blood pressure in patients during extracorporeal circulation. $\mathrm{Med} \mathrm{Sci}$ Monit. 2007; 13:Cr548-54.

18. Jarosz B, Dabrowski W, Marciniak A, Wacinski P, Rzecki Z, Kotlinska E, et al Increase in intra-abdominal pressure raises brain venous pressure, leads to brain ischaemia and decreases brain magnesium content. Magnes Res. 2012;25:89-98.

19. Ameloot K, Genbrugge C, Meex I, Eertmans W, Jans F, De Deyne C, et al. Is venous congestion associated with reduced cerebral oxygenation and worse neurological outcome after cardiac arrest? Crit Care. 2016;20:146.

20. Nguyen DN, Huyghens L, Parra J, Schiettecatte J, Smitz J, Vincent JL. Hypotension and a positive fluid balance are associated with delirium in patients with shock. PLoS One. 2018;13:e0200495.

21. Williams JB, Peterson ED, Wojdyla D, Harskamp R, Southerland KW Ferguson TB, et al. Central venous pressure after coronary artery bypass surgery: does it predict postoperative mortality or renal failure? J Crit Care. 2014;29: 1006-10.

22. Figg KK, Nemergut EC. Error in central venous pressure measurement. Anesth Analg. 2009;108:1209-11.

23. van der Jagt M. Fluid management of the neurological patient: a concise review. Crit Care. 2016;20:126.

24. Denault AY, Azzam MA, Beaubien-Souligny W. Imaging portal venous flow to aid assessment of right ventricular dysfunction. Can J Anaesth. 2018;65:1260-1.

25. Corradi F, Via G, Tavazzi G. What's new in ultrasound-based assessment of organ perfusion in the critically ill: expanding the bedside clinical monitoring window for hypoperfusion in shock. Intensive Care Med. 2020;46:775-9.

26. McNaughton DA, Abu-Yousef MM. Doppler US of the liver made simple. Radiographics. 2011;31:161-88.

27. Styczynski G, Milewska A, Marczewska M, Sobieraj P, Sobczynska M, Dabrowski M, et al. Echocardiographic correlates of abnormal liver tests in patients with exacerbation of chronic heart failure. J Am Soc Echocardiogr 2016;29:132-9.

28. Eljaiek R, Cavayas YA, Rodrigue E, Desjardins G, Lamarche Y, Toupin F, et al High postoperative portal venous flow pulsatility indicates right ventricular dysfunction and predicts complications in cardiac surgery patients. Br J Anaesth 2019;122:206-14.

29. Beaubien-Souligny W, Benkreira A, Robillard P, Bouabdallaoui N, Chassé M, Desjardins G, et al. Alterations in portal vein flow and intrarenal venous flow are associated with acute kidney injury after cardiac surgery: a prospective observational cohort study. J Am Heart Assoc. 2018;7:e009961.

30. Benkreira A, Beaubien-Souligny W, Mailhot T, Bouabdallaoui N, Robillard P Desjardins G, et al. Portal hypertension is associated with congestive encephalopathy and delirium after cardiac surgery. Can J Cardiol. 2019;35:1134-41. 
TABLE E1. Additional references

Adverse effect of congestion on other organs

Prowle JR, Echeverri JE, Ligabo EV, Ronco C, Bellomo R. Fluid balance and acute kidney injury. Nat Rev Nephrol. 2010;6:107-15.

Sundaram V, Fang JC. Gastrointestinal and liver issues in heart failure. Circulation. 2016;133:1696-703.

Verbrugge FH, Bertrand PB, Willems E, Gielen E, Mullens W, Giri S, et al. Global myocardial oedema in advanced decompensated heart failure. Eur Heart J Cardiovasc Imaging. 2017;18:787-94.

Niebauer J, Volk HD, Kemp M, Dominguez M, Schumann RR, Rauchhaus M, et al. Endotoxin and immune activation in chronic heart failure: a prospective cohort study. Lancet. 1999;353:1838-42.

Valentova M, von Haehling S, Bauditz J, Doehner W, Ebner N, Bekfani T, et al. Intestinal congestion and right ventricular dysfunction: a link with appetite loss, inflammation, and cachexia in chronic heart failure. Eur Heart J. 2016;37:1684-91.

Sakka SG, Klein M, Reinhart K, Meier-Hellmann A. Prognostic value of extravascular lung water in critically ill patients. Chest. 2002;122:2080-6.

Madias JE. Apparent amelioration of bundle branch blocks and intraventricular conduction delays mediated by anasarca. J. Electrocardiol. 2005;38:160-5.

Cerebral congestion in other settings

Ameloot K, Genbrugge C, Meex I, Eertmans W, Jans F, De Deyne C, et al. Is venous congestion associated with reduced cerebral oxygenation and worse neurological outcome after cardiac arrest? Crit Care. 2016;20:146.

Nguyen DN, Huyghens L, Parra J, Schiettecatte J, Smitz J, Vincent JL. Hypotension and a positive fluid balance are associated with delirium in patients with shock. PLoS One. 2018;13:e0200495.

Warrillow SJ, Weinberg L, Parker F, Calzavacca P, Licari E, Aly A, et al. Perioperative fluid prescription, complications and outcomes in major elective open gastrointestinal surgery. Anaesth Intensive Care. 2010;38:259-65. 